\title{
Higher Numbers of Pregnancies Associated With an Increased Prevalence of Gestational Diabetes Mellitus: Results From the Healthy Baby Cohort Study
}

\author{
Bingqing Liu ${ }^{1}$, Lulu Song ${ }^{1}$, Lina Zhang ${ }^{1}$, Lulin Wang ${ }^{1}$, Mingyang $\mathrm{Wu}^{1}$, \\ Shunqing $\mathrm{Xu}^{2}$, Zhongqiang $\mathrm{Cao}^{3}$, and Youjie Wang ${ }^{1,2}$
}

\begin{abstract}
${ }^{1}$ Department of Maternal and Child Health, School of Public Health, Tongji Medical College, Huazhong University of Science and Technology, Hubei, China ${ }^{2}$ Key Laboratory of Environment and Health, Ministry of Education \& Ministry of Environmental Protection, and State Key Laboratory of Environmental Health, School of Public Health, Tongji Medical College, Huazhong University of Science and Technology, Hubei, China

${ }^{3}$ Wuhan Children's Hospital (Wuhan Maternal and Child Healthcare Hospital), Tongji Medical College, Huazhong University of Science and Technology, Hubei, China
\end{abstract}

Received November 26, 2018; accepted April 1, 2019; released online April 20, 2019

\begin{abstract}
Background: Pregnancy leads to substantial maternal metabolic and lifestyle alterations. However, it is still unclear whether repeated exposure to these changes will influence the development of gestational diabetes mellitus (GDM). In the present study, we aimed to investigate the association between the number of pregnancies and GDM among Chinese women.

Methods: A total of 7,008 subjects from the Healthy Baby Cohort study were included in this study. The number of pregnancies was classified into three categories: 1,2 , or $\geq 3$ pregnancies. GDM was diagnosed using International Association of Diabetes and Pregnancy Study Groups criteria. Multivariate logistic regression models were used.

Results: In the fully adjusted model, women with $\geq 3$ pregnancies had a 1.27 -fold (95\% confidence interval [CI], 1.05-1.54) higher risk of GDM. Among women $\geq 30$ years old, 2 and $\geq 3$ pregnancies were associated with a higher risk of GDM (odds ratio [OR] 1.32; 95\% CI, 1.01-1.73 and OR 1.54; 95\% CI, 1.17-2.01, respectively). Among women with a pre-pregnancy BMI $<24 \mathrm{~kg} / \mathrm{m}^{2}, \geq 3$ pregnancies were associated with a 1.35 -fold (95\% CI, 1.09-1.67) higher risk of GDM.

Conclusions: Our findings suggested that higher numbers of pregnancies is an independent risk factor of GDM. The association between number of pregnancies and GDM was more prominent among women who were $\geq 30$ years old or with a pre-pregnancy BMI $<24 \mathrm{~kg} / \mathrm{m}^{2}$.
\end{abstract}

Key words: gestational diabetes mellitus; number of pregnancy; cohort study

Copyright $\odot 2019$ Bingqing Liu et al. This is an open access article distributed under the terms of Creative Commons Attribution License, which permits unrestricted use, distribution, and reproduction in any medium, provided the original author and source are credited.

\section{INTRODUCTION}

Gestational diabetes mellitus (GDM) is defined as any degree of glucose intolerance that begins or is recognized for the first-time during pregnancy. ${ }^{1}$ According to the International Association of Diabetes and Pregnancy Study Groups (IADPSG) criteria, GDM constitutes $17.8 \%$ (range, 9.3-25.5\%) of all pregnancies ${ }^{2}$ and has long-lasting health consequences for both mothers and their offspring. GDM increases the maternal risk of developing diabetes, and the offspring's risk of macrosomia, obesity, glucose intolerance, and diabetes. ${ }^{3,4}$ Thus, it is important to identify women at risk of GDM, who would benefit from early preventative strategies. Previously, several GDM risk factors have been identified, including increasing maternal age, obesity, and a family history of diabetes. ${ }^{1}$ However, notable gaps remain in our understanding of risk factors and the pathogenesis of GDM.

Pregnancy leads to substantial maternal metabolic and lifestyle alterations, including decreased insulin sensitivity, an accumu- lation of body fat, body fat central redistribution, decreased physical activity, and increased calorie intake, ${ }^{5-7}$ which are associated with higher risk of GDM. ${ }^{8,9}$ However, it is still unclear whether repeated exposure to these metabolic and lifestyle changes influences the development of GDM in future pregnancies. Various studies have investigated the association between parity and GDM, with conflicting results. ${ }^{10-13}$ However, none of these studies took abortion or stillbirth into consideration. Spontaneous miscarriage occurs in approximately $15 \%$ of clinically recognized pregnancies. ${ }^{14}$ In China, approximately 10 million women received an induced abortion in 2015, ${ }^{15}$ and approximately $50 \%$ of women who underwent an induced abortion experienced repeated abortions. ${ }^{16}$ Therefore, a significant proportion of women might have a high number of pregnancies, despite having a relatively low parity. Because the metabolic alterations caused by pregnancy were observed as early as the first trimester, ${ }^{17}$ abortions and stillbirth might also be accompanied by metabolic alterations lead by pregnancy. Thus, it 
is necessary to investigate whether a high number of pregnancies is associated with GDM risk.

In the present study, we investigated the association between the number of pregnancies and GDM among Chinese women. Our hypothesis was that women with a higher number of pregnancies were at higher risk of developing GDM than women with one pregnancy.

\section{MATERIAL AND METHODS}

\section{Participants}

The study subjects were from Healthy-Baby Cohort-Wuhan (HBC-Wuhan), which is a prospective cohort conducted to explore the environmental and genetic factors that affect health and development. This study recruited 11,311 pregnant women, who came for their first antenatal care visit or birth delivery at Wuhan Children's Hospital (Wuhan Maternal and Child Healthcare Hospital), from September 2012 to October 2014. After excluding those women with a history of diabetes $(n=6)$ and had no available information on fasting, 1-h, or 2-h plasma glucose data $(n=4,297), 7,008$ women were included in the final analysis.

This study was approved by the Medical Ethics Committee of School of Public Health, Tongji Medical School and the Wuhan Children's Hospital (Wuhan Maternal and Child Healthcare Hospital). Signed informed consent was obtained from all participants.

\section{Assessment of GDM}

Participants underwent a 75-g oral glucose tolerance test (OGTT) at 24-28 weeks of gestation. Plasma glucose levels were measured using the Roche Modular P800 automated biochemistry analyzer (Roche Diagnostics USA, Indianapolis, IN, USA) at the Wuhan Children's Hospital (Wuhan Maternal and Child Healthcare Hospital). GDM was diagnosed using the diagnostic criterion of the IADPSG. ${ }^{18}$ Women whose $75 \mathrm{~g}$-OGTT plasma glucose level met one or more of the following criterions were defined as having GDM: fasting plasma glucose level $\geq 5.1$ $\mathrm{mmol} / \mathrm{L}$; 1-h plasma glucose level $\geq 10.0 \mathrm{mmol} / \mathrm{L}$; 2-h plasma glucose level $\geq 8.5 \mathrm{mmol} / \mathrm{L}$.

\section{Assessment of pregnancy}

Information on the number of pregnancies was extracted from medical record of Wuhan Children's Hospital (Wuhan Maternal and Child Healthcare Hospital). Number of pregnancies was classified into three categories: 1,2 , or $\geq 3$ pregnancies.

\section{Assessment of covariates}

In this study, we collected demographic information (age, annual household income, and education background) and lifestyle information (cigarette smoking, passive smoking exposure, and alcohol drinking) via structured questionnaire. All the participants were interviewed face-to-face by trained nurses during delivery hospitalization. Pre-pregnancy weight was self-reported at the first antenatal care visit (at the first trimester), while measured standing height and the results of laboratory were abstracted from medical records. Pre-pregnancy body mass index (BMI) was calculated as weight $(\mathrm{kg})$ divided by the height squared $\left(\mathrm{m}^{2}\right)$.

\section{Statistical analysis}

Categorical and numerical variables were summarized as proportion (\%) and means (standard deviation [SD]), respectively.
Differences in means and proportions between GDM and nonGDM groups were analyzed with analysis of variance (ANOVA) for numerical variables and Chi-square test for categorical variables. A series of logistic regression models were employed to assess the association between number of pregnancies and GDM. Women with one pregnancy were used as the reference group. Model 1 was used to summarize the crude odds ratios without adjustment of any covariates. Model 2 was adjusted for pre-pregnancy BMI. Model 3 was the fully-adjusted model, in which we adjusted maternal age (continuous), pre-pregnancy BMI (continuous), education (middle school or below, high school, or college or above), annual household income $(<50,000$, $50,000-100,000$, or $\geq 100,000$ Yuan), smoking status (current/ ever smoker or never smoker), drinking status (yes or no), and passive smoking (yes or no).

Because increasing age and pre-pregnancy BMI were acknowledged risk factors of GDM, ${ }^{1}$ we further employed subgroup analysis to assess the potential effect modifications by age and pre-pregnancy BMI. SAS 9.4 (SAS Institute Inc, Cary, NC, USA) was used for all analysis. Statistical significance were determined using a two-side probability set at $P<0.05$. For the test of interactive effect, statistical significance was set at a two-side probability of $P<0.1 .^{19}$

\section{RESULTS}

Characteristics of the participants are shown in Table 1. Among 7,008 participants, a total of 1,030 women (14.7\%) were diagnosed as having GDM based on the diagnostic criterion suggested by IADPSG. Women had GDM were significantly older $(P<0.001)$, fatter before pregnancy $(P<0.001)$, and and had lower education background $(P<0.001)$.

We used three logistic regression models to assess the associations between number of pregnancy and GDM. In the

Table 1. Characteristics of the participants according to gestational diabetes

\begin{tabular}{|c|c|c|c|}
\hline \multirow[b]{2}{*}{ Characteristics } & \multicolumn{2}{|l|}{ GDM } & \multirow[t]{2}{*}{$P$} \\
\hline & $\begin{array}{l}\text { Yes } \\
N=1,030\end{array}$ & $\begin{array}{l}\text { No } \\
N=5,978\end{array}$ & \\
\hline Age, years, mean (SD) & $29.6(3.9)$ & $28.3(3.4)$ & $<0.001$ \\
\hline Pre-pregnancy BMI, $\mathrm{kg} / \mathrm{m}^{2}$, mean (SD) & $21.5(3.1)$ & $20.6(2.7)$ & $<0.001$ \\
\hline \multicolumn{4}{|l|}{ Education } \\
\hline Middle school or below (\%) & $132(12.8)$ & $514(8.6)$ & $<0.001$ \\
\hline High school (\%) & $193(18.7)$ & $1012(16.9)$ & \\
\hline College or above (\%) & $705(68.4)$ & $4451(74.5)$ & \\
\hline \multicolumn{4}{|l|}{ Annual household income (Yuan)* } \\
\hline$<50,000(\%)$ & $421(41.9)$ & $2311(39.3)$ & 0.130 \\
\hline $50,000-100,000(\%)$ & $417(41.5)$ & 2459 (41.8) & \\
\hline$>100,000(\%)$ & $167(16.6)$ & $1117(19.0)$ & \\
\hline \multicolumn{4}{|l|}{ Cigarette smoking status } \\
\hline Current or ever smokers (\%) & $9(0.9)$ & $30(0.5)$ & 0.138 \\
\hline Never smokers $(\%)$ & $1021(99.1)$ & $5948(99.5)$ & \\
\hline \multicolumn{4}{|l|}{ Passive smoking exposure } \\
\hline Yes $(\%)$ & $211(20.5)$ & $1274(21.3)$ & 0.549 \\
\hline No $(\%)$ & $819(79.5)$ & $4704(78.7)$ & \\
\hline \multicolumn{4}{|l|}{ Alcohol drinking } \\
\hline Yes (\%) & $22(2.1)$ & $129(2.2)$ & 0.964 \\
\hline No $(\%)$ & $1008(97.9)$ & $5849(97.8)$ & \\
\hline
\end{tabular}

BMI, body mass index; GDM, gestational diabetes mellitus; SD, standard deviation.

*One Yuan RMB is equal to 0.1462 United States dollars. 

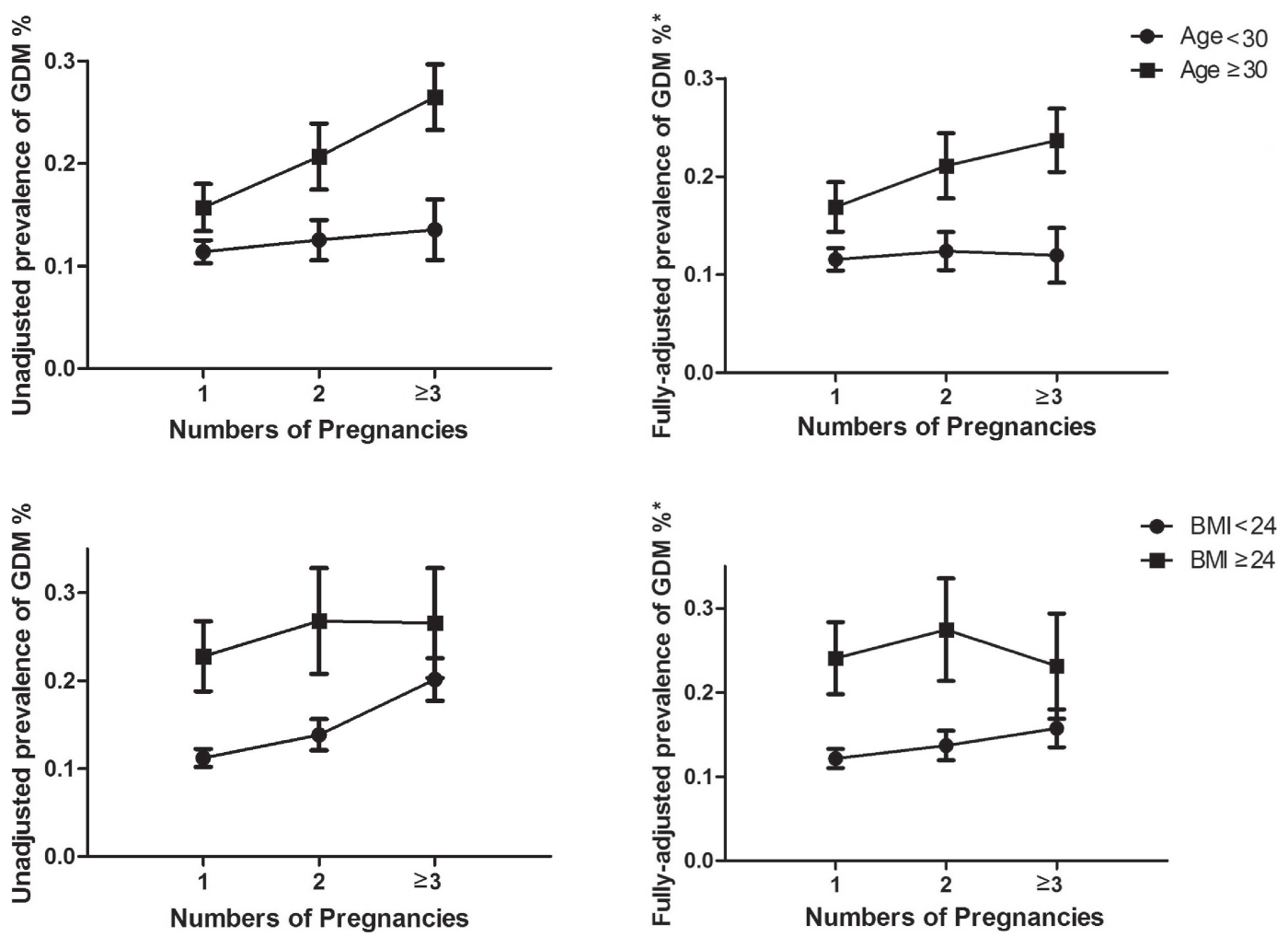

Figure 1. Prevalence of gestational diabetes in different age and pre-pregnancy body mass index groups according to number of pregnancies. ${ }^{*}$ The adjusted covariates included age, pre-pregnancy BMI, education, annual household income, smoking status, drinking status and passive smoking exposure.

unadjusted model, women who had 2 and $\geq 3$ pregnancies had 1.29 (95\% CI, 1.10-1.51) and 1.89 (95\% CI, 1.60-2.23) times higher risk of GDM than women who had one pregnancy. After adjusting for age, pre-pregnancy BMI, education, annual household income, smoking status, drinking status, and passive smoking exposure, we found that $\geq 3$ pregnancies was still associated with higher risk of GDM (OR 1.27; 95\% CI, 1.05-1.54).

We further did stratified analysis by age and pre-pregnancy BMI. In the fully-adjusted model, 2 and $\geq 3$ pregnancies were associated with higher risk of GDM (OR 1.32; 95\% CI, 1.01-1.73 and OR $1.54 ; 95 \% \mathrm{CI}, 1.17-2.01$, respectively) in women $\geq 30$ years old ( $P_{\text {for interaction }}=0.048$; Figure 1 and Table 2$)$. After adjusted potential confounders, $\geq 3$ pregnancies were associated with higher risk of gestational diabetes (OR 1.35; 95\% CI, 1.091.67 ) in women with pre-pregnancy BMI $<24 \mathrm{~kg} / \mathrm{m}^{2}$ (Figure 1 and Table 2). However, we did not find significant interactions between pre-pregnancy BMI and number of pregnancy on GDM $\left(P_{\text {for interaction }}=0.591\right)$.

We compared the main characteristics between women who were included and excluded. Except for drinking status, the main characteristics of the included and excluded participants, including number of pregnancy (1.7 vs. 1.9), age at delivery (28.5 vs. 27.8 years), pre-pregnancy BMI (20.7 vs. $\left.20.3 \mathrm{~kg} / \mathrm{m}^{2}\right)$, gestational weight gain $(17.3$ vs. $16.9 \mathrm{~kg})$, and passive smoking exposure (21.2\% vs. $23.9 \%)$ were statistically different.

\section{DISCUSSION}

In this study, we found that women with $\geq 3$ pregnancies had a 1.27 -fold (95\% CI, 1.05-1.54) higher risk of developing GDM, while 2 pregnancies was not associated with GDM. These
Table 2. Odd Ratios and $95 \%$ confidence intervals for GDM according to number of pregnancies

\begin{tabular}{|c|c|c|c|c|}
\hline $\begin{array}{l}\text { Number of } \\
\text { pregnancy }\end{array}$ & $\begin{array}{l}\text { Number of } \\
\text { subjects }\end{array}$ & $\begin{array}{l}\text { Model 1: } \\
\text { unadjusted }\end{array}$ & $\begin{array}{l}\text { Model 2: model } \\
\text { 1+BMI }\end{array}$ & $\begin{array}{l}\text { Model 3: fully- } \\
\text { adjusted model* }\end{array}$ \\
\hline \multicolumn{5}{|c|}{ Total population } \\
\hline 1 & 4,060 & 1 & 1 & 1 \\
\hline 2 & 1,698 & $1.29(1.10,1.51)$ & $1.27(1.08,1.49)$ & $1.16(0.98,1.37)$ \\
\hline$\geq 3$ & 1,250 & $1.89(1.60,2.23)$ & $1.79(1.51,2.11)$ & $1.27(1.05,1.54)$ \\
\hline \multicolumn{5}{|c|}{ Age $<30$ years } \\
\hline 1 & 3,091 & 1 & 1 & 1 \\
\hline 2 & 1,093 & $1.12(0.90,1.38)$ & $1.11(0.90,1.38)$ & $1.08(0.87,1.35)$ \\
\hline$\geq 3$ & 517 & $1.22(0.93,1.61)$ & $1.19(0.90,1.57)$ & $1.04(0.77,1.39)$ \\
\hline \multicolumn{5}{|c|}{ Age $\geq 30$ years } \\
\hline 1 & 969 & 1 & 1 & 1 \\
\hline 2 & 605 & $1.40(1.08,1.82)$ & $1.38(1.06,1.80)$ & $1.32(1.01,1.73)$ \\
\hline$\geq 3$ & 733 & $1.94(1.52,2.46)$ & $1.89(1.49,2.40)$ & $1.54(1.17,2.01)$ \\
\hline \multicolumn{5}{|c|}{ BMI $<24 \mathrm{~kg} / \mathrm{m}^{2}$} \\
\hline 1 & 3631 & 1 & 1 & 1 \\
\hline 2 & 1487 & $1.27(1.06,1.53)$ & $1.27(1.06,1.52)$ & $1.15(0.95,1.38)$ \\
\hline$\geq 3$ & 1058 & $2.00(1.66,2.40)$ & $1.94(1.62,2.33)$ & $1.35(1.09,1.67)$ \\
\hline \multicolumn{5}{|c|}{$\mathrm{BMI} \geq 24 \mathrm{~kg} / \mathrm{m}^{2}$} \\
\hline 1 & 426 & 1 & 1 & 1 \\
\hline 2 & 209 & $1.24(0.85,1.82)$ & $1.25(0.85,1.83)$ & $1.20(0.81,1.78)$ \\
\hline$\geq 3$ & 192 & $1.23(0.83,1.82)$ & $1.23(0.83,1.82)$ & $0.95(0.60,1.50)$ \\
\hline
\end{tabular}

BMI, body mass index.

*fully-adjusted model: adjusted for age, pre-pregnancy BMI, education background, annual household income, smoking status, drinking status and passive smoking exposure.

associations were more prominent among women aged $\geq 30$ years old or women with pre-pregnancy BMI $<24 \mathrm{~kg} / \mathrm{m}^{2}$.

There have been few studies on the association between the number of pregnancies and GDM, some of them have conflicting results. A study conducted among 11,205 women found that $\geq 3$ 
live births increased the risk of GDM in white, black, and Southeast Asian women. ${ }^{12}$ Another study found that multiparity was associated with a higher risk of GDM. ${ }^{13}$ These two studies support our current findings. Inconsistent with these two studies, Xiong et al found that multiparity was not associated with GDM..$^{11}$ It should be noted that these studies used different criteria to diagnose GDM, which might at least partially explain the discrepancy in the results. Moreover, these studies only investigated the association between the number of live births and GDM, while abortions and stillbirth, which are also accompanied by metabolic alterations lead by pregnancy, ${ }^{17}$ were not considered.

The mechanism underlying the link between the number of pregnancies and GDM is unclear. During pregnancy, the increased secretion of steroids and peptide hormones leads to a progressive rise in maternal tissue insulin resistance. ${ }^{6}$ Although glucose homeostasis is restored to preconception levels shortly after delivery, ${ }^{20}$ repeated exposure to these drastic hormonal and metabolic changes may still pathologically perturb glucose metabolism. Lifestyle changes caused by pregnancy may also increase the risk of GDM among women with a high number of pregnancies. Pregnant women tend to reduce their physical activity and increase their calorie intake during and after pregnancy, $5,21,22$ especially in China, where women are encouraged to rest in bed and consume high-calorie food during pregnancy and the postpartum period. ${ }^{23}$ It is well established that a high-calorie diet induces insulin resistance, while physical inactivity before and during pregnancy is associated with GDM. ${ }^{24,25}$ These unhealthy changes in lifestyle may have longterm metabolic effects that extend into future pregnancies. Besides, those pregnancy-related unhealthy lifestyles might increase gestational weight gain during the next pregnancy, which is a risk factor of GDM, ${ }^{26}$ leading to the hypothesis that the association between number of pregnancy and GDM might be mediated by gestational weight gain. However, gestational weight gain was not significantly different among pregnant women with 1,2 , or $\geq 3$ pregnancies in the present study $(P=0.427)$, which suggests that gestational weight gain was not a mediator for the relationship between number of pregnancy and GDM. Thus, gestational weight gain could probably not account for the observed positive association between the number of pregnancies and GDM in the current study.

In this study, we found that the association between the number of pregnancies and GDM was more prominent among women aged $\geq 30$ years. The rate of impaired glucose tolerance and impaired fasting glucose was previously found to increase with age among women. ${ }^{27}$ This could be attributed to the reduced physical activity and muscle mass reported in older women. ${ }^{28,29}$ Physical inactivity leads to a decline in glucose tolerance and an increase in fasting blood glucose, ${ }^{30,31}$ while skeletal muscle mass was reported to negatively predict 2-hour plasma glucose levels in subjects with normal glucose tolerance. ${ }^{32}$ These age-related changes in glucose metabolism would be exacerbated by pregnancy, which may explain the stronger association between the number of pregnancies and GDM among women aged $\geq 30$ years.

We also found that, although overweight/obesity women had higher GDM prevalence, the association between the number of pregnancies and GDM was more prominent among lean women. However, it should be noted that the interaction between prepregnancy BMI and number of pregnancies on GDM was not significant, which indicated that this BMI-specific association between number of pregnancies and GDM might be attributed to chance. The pre-pregnancy BMI-stratified analyses should be considered as exploratory. It's reported that obese women demonstrate less weight gain and smaller rises in fasting plasma glucose during pregnancy. ${ }^{33}$ Thus, pregnancies appear to have less effect on glucose homeostasis in obese women. Therefore, although the obesity/overweight women had higher GDM prevalence, the association between the number of pregnancies and GDM was not significant.

The present study has several strengths. It had a relatively large sample size, and OGTT measurements were performed using the same instruments at one hospital, which decreased detection bias. However, it has a number of limitations. First, the relatively large number of subjects excluded from the study might have introduced selection bias. However, although there were significant differences between subjects who were included and excluded, the differences in major variables between the two groups were relatively small. Because of the large sample of the inclusion and exclusion, a slight difference would lead to a statistically significant difference. Second, number of pregnancies was obtained from medical records, but it was self-reported to doctors during the first prenatal visit. Therefore, recall bias might have occurred. Moreover, information about abortions and stillbirths is considered private in China, so the numbers of these pregnancies might be under-reported, which might lead to an under-estimation of the association between the number of pregnancies and GDM. Third, our study participants were all Chinese women, which minimized the confounding effects by ethnic background but might reduce the extension of our results to non-Asian populations. Fourth, we cannot control for unmeasured confounding, such as family history of diabetes and diet. Further studies with this information are needed to confirm the finding of this study.

In summary, we found that higher number of pregnancies was independently associated with a higher risk of GDM, particularly among women aged $\geq 30$ years old and women with prepregnancy BMI $<24 \mathrm{~kg} / \mathrm{m}^{2}$. These findings suggest that clinical practitioners should take the number of pregnancies into consideration while assessing GDM risk. Further prospective studies are needed to confirm our findings and to elucidate the mechanism underlying the association between the number of pregnancies and GDM.

\section{ACKNOWLEDGMENTS}

We thank all the study participants and the entire HBC Study team for their generous help.

Funding: This work was supported by the National Natural Science Foundation of China (grant numbers: 81273083, 91643207), Hubei Province Health \& Family Planning Scientific Research Project (grant number: WJ2017Z001), Wuhan Municipal Health and Family Planning Commission Scientific Grant (grant number: WG16B01), and Health Commission of Hubei Province scientific research project (Grant number: WJ2019Q003).

Contribution to authorship: Y.W., Z. C. and B.L. conceived and designed the study. L.S. and L.Z. assisted with study design. B.L. contributed to statistical analysis and wrote the manuscript. L.W., M.W. and S.X. provided statistical advice and assisted with data analysis. Y.W. reviewed and edited the manuscript. All authors approved the final version to be published.

Conflicts of interest: None declared. 


\section{REFERENCES}

1. American Diabetes Association. Gestational diabetes mellitus. Diabetes Care. 2004;27(Suppl 1):S88-S90.

2. Sacks DA, Hadden DR, Maresh M, et al; HAPO Study Cooperative Research Group. Frequency of gestational diabetes mellitus at collaborating centers based on IADPSG consensus panel-recommended criteria: the Hyperglycemia and Adverse Pregnancy Outcome (HAPO) Study. Diabetes Care. 2012;35:526-528.

3. Bellamy L, Casas JP, Hingorani AD, Williams D. Type 2 diabetes mellitus after gestational diabetes: a systematic review and metaanalysis. Lancet. 2009;373:1773-1779.

4. Reece EA, Leguizamón G, Wiznitzer A. Gestational diabetes: the need for a common ground. Lancet. 2009;373:1789-1797.

5. Crozier SR, Robinson SM, Godfrey KM, Cooper C, Inskip HM. Women's dietary patterns change little from before to during pregnancy. J Nutr. 2009;139:1956-1963.

6. Lain KY, Catalano PM. Metabolic changes in pregnancy. Clin Obstet Gynecol. 2007;50:938-948.

7. Pereira MA, Rifas-Shiman SL, Kleinman KP, Rich-Edwards JW, Peterson KE, Gillman MW. Predictors of change in physical activity during and after pregnancy: Project Viva. Am J Prev Med. 2007;32:312-319.

8. Chu SY, Callaghan WM, Kim SY, et al. Maternal obesity and risk of gestational diabetes mellitus. Diabetes Care. 2007;30:2070-2076.

9. Tobias DK, Zhang C, van Dam RM, Bowers K, Hu FB. Physical activity before and during pregnancy and risk of gestational diabetes mellitus: a meta-analysis. Diabetes Care. 2011;34:223-229.

10. Solomon CG, Willett WC, Carey VJ, et al. A prospective study of pregravid determinants of gestational diabetes mellitus. JAMA. 1997; 278:1078-1083

11. Xiong X, Saunders LD, Wang FL, Demianczuk NN. Gestational diabetes mellitus: prevalence, risk factors, maternal and infant outcomes. Int J Gynaecol Obstet. 2001;75:221-228.

12. Dornhorst A, Paterson CM, Nicholls JS, et al. High prevalence of gestational diabetes in women from ethnic minority groups. Diabet Med. 1992;9:820-825.

13. Harris SB, Caulfield LE, Sugamori ME, Whalen EA, Henning B. The epidemiology of diabetes in pregnant Native Canadians. A risk profile. Diabetes Care. 1997;20:1422-1425.

14. Rai R, Regan L. Recurrent miscarriage. Lancet. 2006;368:601-611.

15. National Health and Family Planning Commission of People's Republic of China. China health and family planning statistics yearbook 2015. Peking Union Medical College Press Beijing; 2016 [in Chinese].

16. Cheng Y, Zhou Y. Situation of repeated induced abortion and postabortion care in China. J Int Reprod Health/Fam Plan. 2010;29: 324-326, 332 [in Chinese].

17. Mouzon SH, Lassance L. Endocrine and metabolic adaptations to pregnancy; impact of obesity. Horm Mol Biol Clin Investig. 2015; 24:65-72.

18. International Association of Diabetes and Pregnancy Study Groups
Consensus Panel, Metzger BE, Gabbe SG, Persson B, et al International association of diabetes and pregnancy study groups recommendations on the diagnosis and classification of hyperglycemia in pregnancy. Diabetes Care. 2010;33:676-682.

19. Sun X, Briel M, Walter SD, et al. Is a subgroup effect believable? Updating criteria to evaluate the credibility of subgroup analyses. BMJ. 2010;340:c117.

20. Berggren EK, Presley L, Amini SB, Hauguel-de Mouzon S, Catalano PM. Are the metabolic changes of pregnancy reversible in the first year postpartum? Diabetologia. 2015;58:1561-1568.

21. Schauberger CW, Rooney BL, Brimer LM. Factors that influence weight loss in the puerperium. Obstet Gynecol. 1992;79:424-429.

22. Smith DE, Lewis CE, Caveny JL, Perkins LL, Burke GL, Bild DE. Longitudinal changes in adiposity associated with pregnancy. The CARDIA Study. Coronary Artery Risk Development in Young Adults Study. JAMA. 1994;271:1747-1751.

23. Holroyd E, Twinn S, Yim IW. Exploring Chinese women's cultural beliefs and behaviours regarding the practice of "doing the month". Women Health. 2004;40:109-123.

24. Aune D, Sen A, Henriksen T, Saugstad OD, Tonstad S. Physical activity and the risk of gestational diabetes mellitus: a systematic review and dose-response meta-analysis of epidemiological studies. Eur J Epidemiol. 2016;31:967-997.

25. Bakker LE, van Schinkel LD, Guigas B, et al. A 5-day high-fat, high-calorie diet impairs insulin sensitivity in healthy, young South Asian men but not in Caucasian men. Diabetes. 2014:63:248-258.

26. MacDonald SC, Bodnar LM, Himes KP, Hutcheon JA. Patterns of gestational weight gain in early pregnancy and risk of gestational diabetes mellitus. Epidemiology. 2017;28:419-427.

27. DECODE Study Group. Age- and sex-specific prevalences of diabetes and impaired glucose regulation in 13 European cohorts. Diabetes Care. 2003;26:61-69.

28. Janssen I, Heymsfield SB, Wang ZM, Ross R. Skeletal muscle mass and distribution in 468 men and women aged 18-88 yr. J Appl Physiol (1985). 2000;89:81-88.

29. Zang J, Ng SW. Age, period and cohort effects on adult physical activity levels from 1991 to 2011 in China. Int J Behav Nutr Phys Act. 2016;13:40.

30. Chasan-Taber L, Silveira M, Lynch KE, et al. Physical activity before and during pregnancy and risk of abnormal glucose tolerance among Hispanic women. Diabetes Metab. 2014;40:67-75.

31. Medek H, Halldorsson T, Gunnarsdottir I, Geirsson RT. Physical activity of relatively high intensity in mid-pregnancy predicts lower glucose tolerance levels. Acta Obstet Gynecol Scand. 2016;95:10551062.

32. Rattarasarn C, Leelawattana R, Soonthornpun S. Contribution of skeletal muscle mass on sex differences in 2-hour plasma glucose levels after oral glucose load in Thai subjects with normal glucose tolerance. Metabolism. 2010;59:172-176.

33. Forbes S, Barr SM, Reynolds RM, et al. Convergence in insulin resistance between very severely obese and lean women at the end of pregnancy. Diabetologia. 2015;58:2615-2626. 